Tema: Solidificação / Lingotamento

\title{
CLASSIFICAÇÃO COMPUTACIONAL DA SEGREGAÇÃO CENTRAL DE PLACAS PELO PROCESSAMENTO DE IMAGENS DE IMPRESSÕES DE BAUMANN*
}

\author{
Franz Ramstorfer ${ }^{1}$ \\ Antonio Eli Nunes ${ }^{2}$ \\ Lara Crysthine Paes Ret ${ }^{3}$
}

Resumo

Impressões de Baumann são utilizadas amplamente nas aciarias para a avaliação e monitoramento da qualidade interna de placas: em particular a segregação central. A análise e monitoramento da segregação central é uma base importante do monitoramento da condição da máquina de lingotamento contínuo, da otimização dos parâmetros do processo de lingotamento contínuo e da qualidade das placas. Em geral, o tipo e a intensidade da segregação central são determinados através da comparação da imagem real com imagens de referência do padrão aplicado. $O$ presente trabalho foi focado no desenvolvimento de uma ferramenta de análise computacional, precisa e fácil de usar pelos operadores do laboratório de Baumann. O objetivo foi estabelecer uma base padronizada e confiável para o processo de monitoramento da segregação central. A metodologia desenvolvida é baseada em processamento de imagem computacional de uma impressão de Baumann em tons de cinza. Observou-se que a classificação de segregação pode ser realizada com uma precisão excelente através da análise computacional dos dados de imagem da segregação central. O procedimento de classificação foi implementado com a utilização de uma rede neural feed forward.

Palavras-chave: Lingotamento contínuo; Segregação central; Impressões de Baumann; Processamento de imagem; Rede neural.

\section{COMPUTATIONAL CLASSIFICATION OF CENTERLINE SEGREGATION OF SLABS BY IMAGE PROCESSING OF BAUMANN PRINTS}

\begin{abstract}
Baumann prints are widely used in steel making plants for the evaluation and monitoring of the internal quality of slabs: in particular the centerline segregation. The analysis and monitoring of centerline segregation is an important basis of condition monitoring of the caster, the optimization of the continuous casting process and the slab quality. In general, the type and the intensity of centerline segregation are determined by comparing the actual image with reference images of the applied standard. The present work was focused on the development of a precise and easy-to-use computational analyses tool for operators of the Baumann laboratory. The goal was to establish a standardized and trustworthy basis for the monitoring process of the centerline segregation. The developed methodology is based on computational processing of a grayscale image of a Baumann print. It was observed that the segregation classification can be realized with an excellent precision by the computational analyses of the image data. The classification procedure was implemented with the use of a feed forward neural network.
\end{abstract}

Keywords: Continuous slab casting; Centerline segregation; Baumann prints; Image processing; Neural network.

1 Engenheiro Mecânico, Ph.D., Especialista de processos, Usiminas, Cubatão, SP, Brasil.

2 Engenheiro Metalurgista, Gerente de conversores, Usiminas, Cubatão, SP, Brasil.

3 Engenheira Metalurgista, Gerente técnica de aciaria, Usiminas, Cubatão, SP, Brasil.

* Contribuição técnica ao $45^{\circ}$ Seminário de Aciaria - Internacional, 25 a 28 de maio de 2014, Porto Alegre, RS, Brasil. 


\section{INTRODUÇÃo}

Macrosegregação é uma não uniformidade de composição química, que pode ser observada na seção transversal e longitudinal dos produtos lingotados, em particular na linha central ou fora da linha central das placas, chamada segregação central. Macrosegregação é uma característica intrínseca da solidificação de aços. Durante a solidificação, o líquido fica enriquecido de soluto na zona pastosa, pois elementos dissolvidos têm, em geral, uma maior solubilidade na fase líquida do que na fase sólida e, portanto, tendem a sair da solução antes da solidificação final [1]. Estes elementos se difundem em direção ao líquido, mas devido à cinética de solidificação, ficam restritos a uma camada de líquido próxima a fronteira sólido/líquido, dentro da zona pastosa. A causa da macrosegregação no processo de lingotamento é o movimento relativo ou fluxo do líquido segregado durante a solidificação. Estes fluxos do aço líquido são provocados por contração de solidificação, contrações térmicas do líquido e do sólido, flutuabilidade devido aos gradientes térmicos, movimento relativo ou deformação da pele solidificada, entre outros.

Os principais fatores do processo de lingotamento contínuo que influenciam na intensidade da segregação são a composição química do aço, o superaquecimento do aço líquido [2], a velocidade de lingotamento contínuo [3], a intensidade e distribuição do resfriamento secundário [4] e a geometria da máquina (desvios do alinhamento e espaçamento dos rolos de suporte) [5].

A segregação central pode acarretar heterogeneidades nas propriedades mecânicas do aço, além de fragilização e enfraquecimento de seções dos produtos laminados. A forma, distribuição, dimensão e intensidade da segregação central influencia o desempenho do produto final.

As contramedidas para reduzir a severidade da segregação são a agitação eletromagnética posicionada estrategicamente nos segmentos ou veio [2], a previsão precisa e controle de posição do ponto final da solidificação [5], o ajuste e monitoramento da geometria da máquina [6] e redução suave do espaçamento (Soft Reduction) [5].

A análise e monitoramento da segregação central é uma das bases importantes do monitoramento da condição da máquina de lingotamento contínuo, a otimização dos parâmetros do processo de lingotamento contínuo e a qualidade das placas. As técnicas de caracterização da segregação central aplicadas pelas aciarias são qualitativas (impressão metalográfica) [8] ou extremamente precisas (p.ex. análise da distribuição dos elementos químicos ou análise da emissão ótica com espectrômetro) [9]. Estas técnicas avançadas requerem equipamentos muito sofisticados, que não permitem uma avaliação rápida. Apenas as técnicas quantitativas, que representam um gráfico da qualidade interna do material lingotado, permitem um monitoramento de qualidade em uma base diária.

As técnicas de decapagem mais utilizadas para revelar macrosegregação nas impressões metalográficas, são: a impressão de enxofre, o ataque da amostra com persulfato de amônio, ácido clorídrico ou ácido pícrico. Todas estas técnicas utilizam o princípio, que as regiões com níveis de material de segregação mais elevados são sujeitas a ataque químico preferencial. A aplicação específica de uma técnica depende também da composição química do aço. A impressão de enxofre requer, por exemplo, um nível significativo de enxofre, outros agentes atacantes podem exigir níveis mínimos de carbono ou fósforo. Outras questões que influenciam a seleção da técnica de caracterização são a necessidade da velocidade da obtenção do resultado, os custos e a segurança do pessoal. Nas aciarias da Usiminas, a

* Contribuição técnica ao 45 Seminário de Aciaria - Internacional, 25 a 28 de maio de 2014, Porto Alegre, RS, Brasil. 
avaliação da segregação central é realizada através das impressões de Baumann conforme as normas ISO 4968 [10], BS 6285 [11] e ASTM E1180-08 [12]. Além dos outros possíveis defeitos internos das placas, o tipo e a espessura da segregação central são visualmente classificados pelos especialistas através da comparação visual da impressão de Baumann da amostra com imagens de referência do padrão aplicado. Nas últimas décadas, vários padrões de classificação da segregação central foram desenvolvidos pelas empresas siderúrgicas conforme as necessidades específicas delas. Os mais conhecidos hoje em dia são os padrões da Nippon Steel Corporation e da Mannesmann.

A classificação manual da segregação central entre os vários níveis de severidade através das imagens de referência depende largamente do nível de experiência, conhecimentos e rigor individual dos especialistas responsáveis. Como uma consequência, sempre existe uma dispersão e insegurança do resultado final da avaliação. A redução do fator humano no processo de avaliação pode ser feita pelo uso da análise computacional. Uma metodologia de análise computacional das impressões de Baumann foi apresentada no trabalho [13] onde a continuidade da segregação central foi analisada pela transformação da imagem em tons de cinza para uma imagem em preto e branco e a aplicação de vários filtros digitais. A continuidade da segregação central (porcentagem) foi calculada pela comparação dos comprimentos das áreas pretas (segregação) e áreas brancas (sem segregação). A espessura da segregação não foi analisada neste trabalho.

O foco do presente trabalho foi o desenvolvimento de uma ferramenta de análise computacional, precisa e fácil de usar pelos especialistas do laboratório de Baumann, que classifica as impressões digitais conforme o padrão aplicado.

\section{MATERIAL E MÉTODOS}

\subsection{Metodologia da Avaliação Atual}

O primeiro passo da preparação das impressões de Baumann nas aciarias da Usiminas é o corte da amostra (a seção transversal da placa) de uma espessura de aprox. $150 \mathrm{~mm}$. Depois cortar, a amostra é usinada para corrigir as irregularidades superficiais. O ataque químico, que revela finalmente a segregação, é realizado através da aplicação superficial de uma solução de persulfato de amônio $\left(\left(\mathrm{NH}_{4}\right)_{2} \mathrm{~S}_{2} \mathrm{O}_{8}\right)$, que ataca a segregação dos elementos $\mathrm{C}, \mathrm{Mn}$ e $\mathrm{P}$ do aço. Este método revela a imagem na própria amostra. A imagem da amostra é capturada através de uma câmera, passando por um processo de digitalização e, em seguida, é disponibilizada na forma de arquivo eletrônico. Esta imagem, em tons de cinza capturada, tem um tamanho de aprox. $5000 \times 500$ pixels. As imagens, que são montadas de várias sub-imagens (Figura 1), são a base de análise deste trabalho.

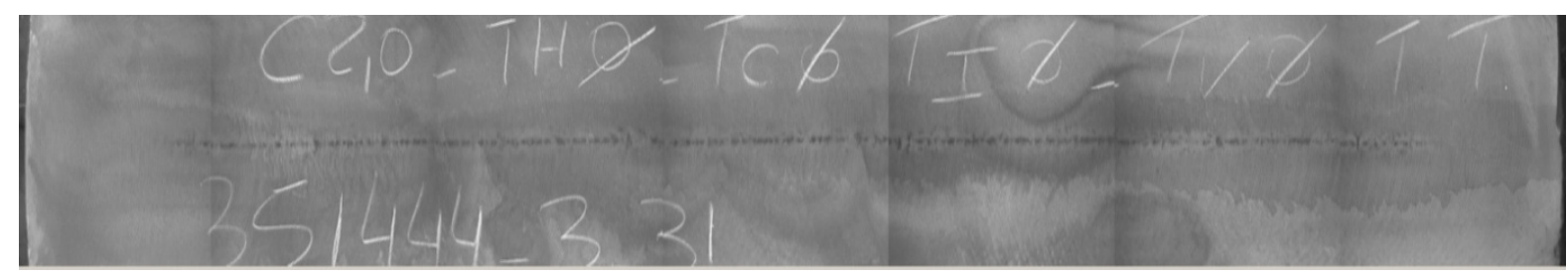

Figura 1. Impressão de Baumann que consiste de sete sub-imagens.

Pode ser visto, na Figura 1, que o brilho não se mantém constante ao longo da largura da imagem. Este fato não é tão importante na avaliação humana da imagem,

* Contribuição técnica ao 45 Seminário de Aciaria - Internacional, 25 a 28 de maio de 2014, 
mas representa um assunto considerável no desenvolvimento do procedimento (algoritmo) de avaliação computacional. Além disso, a superfície mostra várias áreas mais escuras ou claras, causado pelo ataque químico não homogêneo. $O$ procedimento computacional tem que ser suficientemente robusto para ignorar essas deficiências.

Nas aciarias da Usiminas, a segregação central é classificada pelo tipo e espessura da segregação central. Tabela 1 mostra nove exemplos típicos da classificação da segregação central.

Tabela 1. Nove exemplos da classificação da segregação central

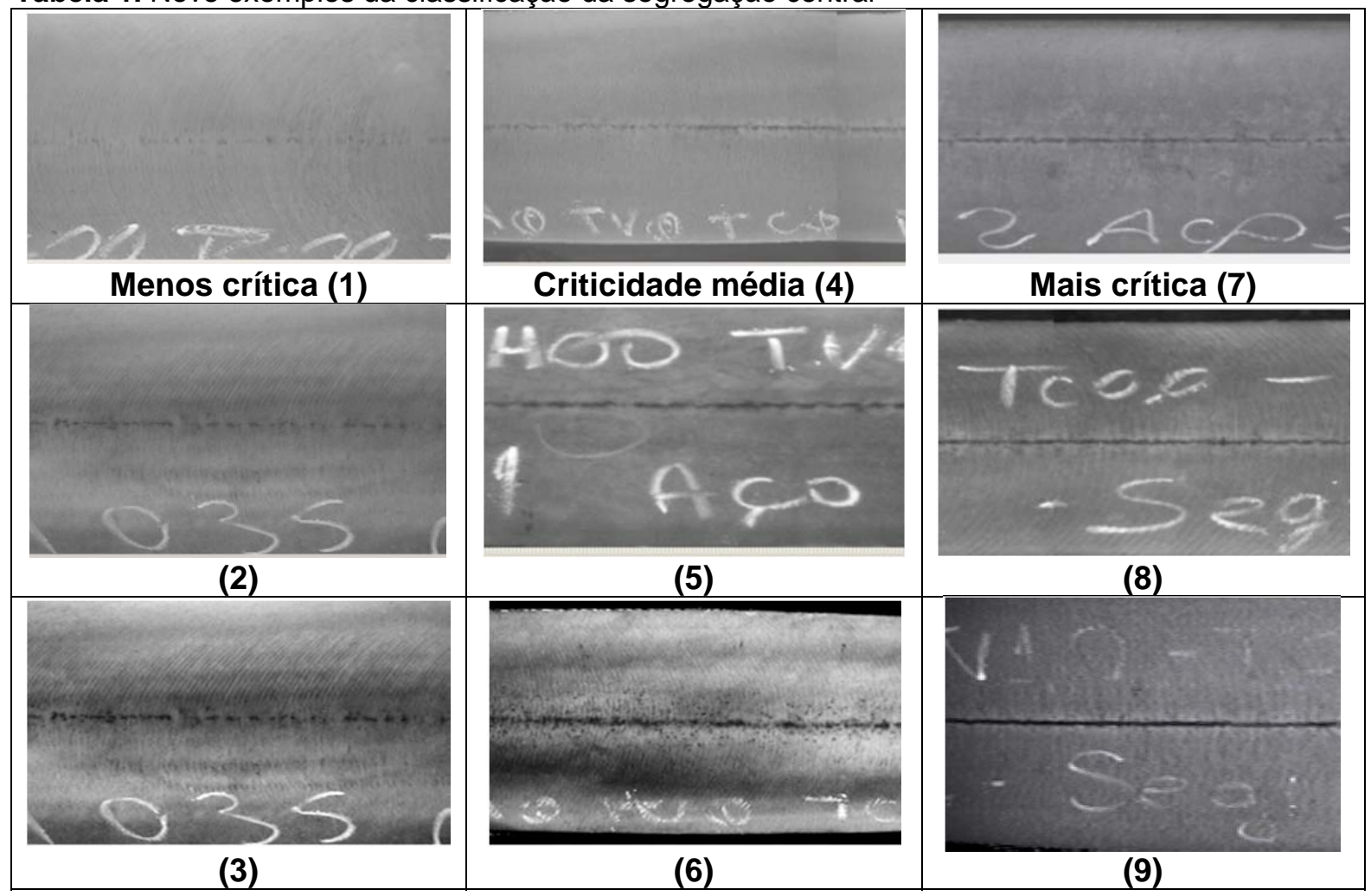

\subsection{Metodologia da Avaliação Computacional}

Como já mencionado anteriormente, a imagem da impressão de Baumann capturada é uma imagem de 8 bits em tons de cinza. Este tipo de imagem permite a gravação das 256 intensidades de tons de cinza diferentes de 0 (ausência total, preto) a 255 (presença total, branco). A imagem é representada por uma matriz bidimensional I(i, j), com um tamanho típico $\mathrm{M} \times \mathrm{N}$ de aprox. $5000 \times 500$ itens. Uma representação gráfica desta matriz é mostrada na Figura 2.

O primeiro passo do processo de análise é a localização (marcação) automática ou manual dos pixels, que fazem parte da linha da segregação central. Normalmente, a linha da segregação central está locada dentro de uma faixa de 5 a 15 pixels.

* Contribuição técnica ao 45 Seminário de Aciaria - Internacional, 25 a 28 de maio de 2014, 


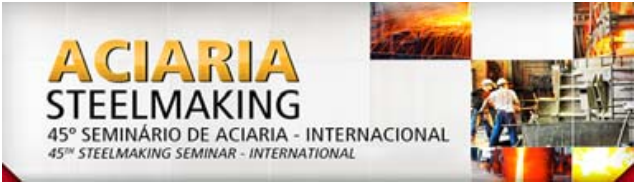

$(0,0) \stackrel{i}{\longrightarrow}$

$(\mathrm{M}, 0)$

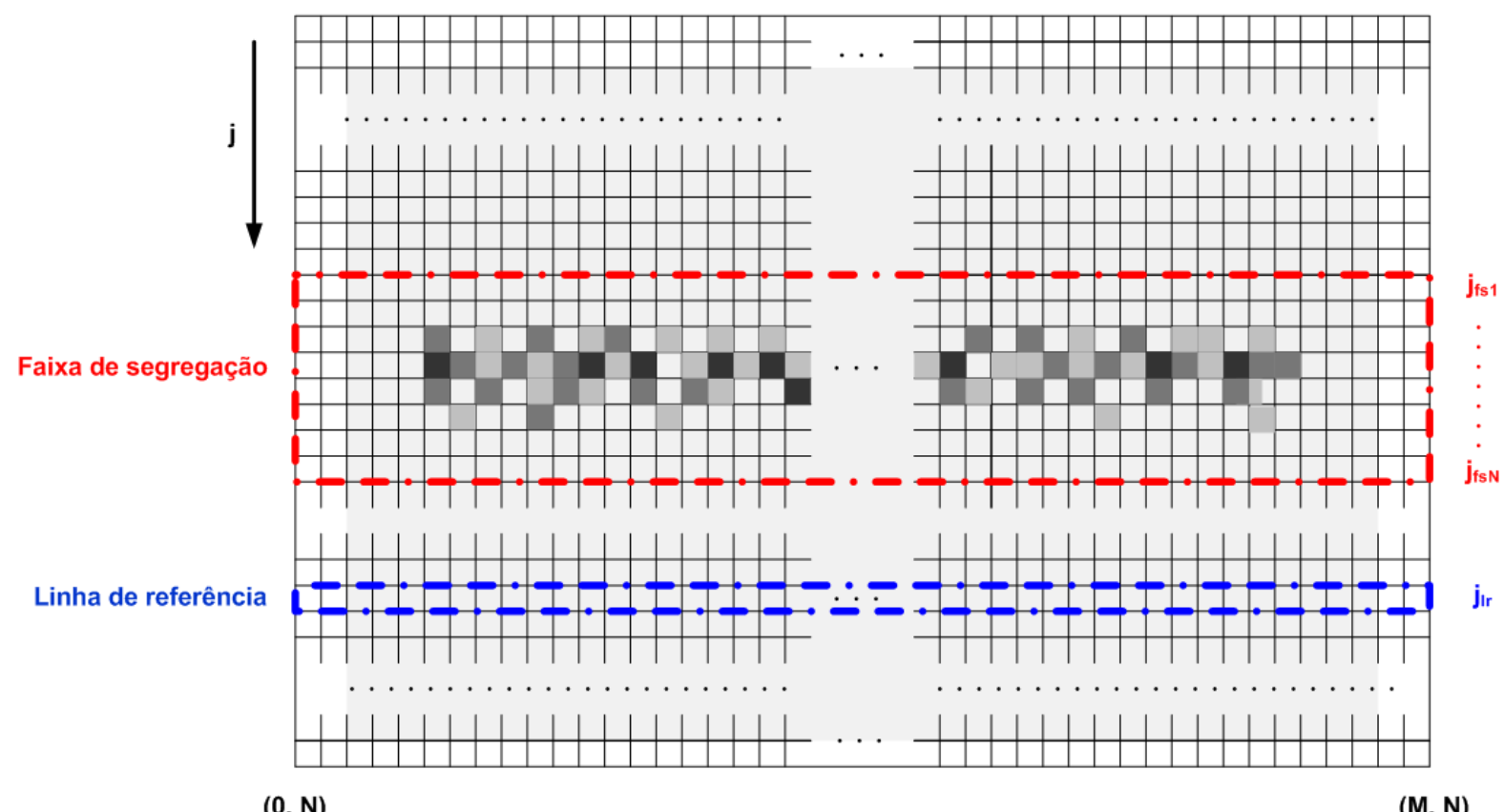

Figura 2. Representação da impressão de Baumann em uma matriz bidimensional.

Para transformar a informação da segregação bidimensional (a faixa da segregação) para uma função unidimensional da largura, foi calculado o valor do pixel mais escuro de cada coluna ( $\left(, j_{\mathrm{fs} 1}-\mathrm{j}_{\mathrm{fs} N}\right)$ da faixa da segregação central:

$$
I_{S C}(i)=\operatorname{Mín}\left(\left.I(i, j)\right|_{j=j_{\mathrm{fs} 1}} ^{j=j_{\text {fNN }}}\right) \text {. }
$$

A avaliação da segregação central não pode ser simplesmente realizada através do valor de intensidade absoluto, Isc(i), pois cada imagem mostra uma iluminação ligeiramente diferente e, além disso, a intensidade da iluminação também varia com a posição. Por isso, a função da intensidade da segregação, Isc(i) é comparada com uma linha de intensidade de referência, $\mathrm{I}_{\mathrm{R}}\left(\mathrm{i}, \mathrm{j}_{\mathrm{I}}\right)$. Descobriu-se que é vantajoso escolher uma linha de referência paralela, localizada fora da área de segregação. $A$ distância vertical entre a linha de segregação e a linha de referência deveria ser escolhida entre 10 e 70 pixels. A intensidade de segregação relativa, ISC_R, é calculada pela diferença entre o valor de tom de cinza da linha de referência, I(i, $\left.j_{\text {Ir }}\right)$, e a função de intensidade da segregação $I_{S c}(i)$ :

$$
\mathrm{I}_{\mathrm{SC} \_\mathrm{R}}(\mathrm{i})=\mathrm{I}\left(\mathrm{i}, \mathrm{j}_{\mathrm{lr}}\right)-\mathrm{I}_{\mathrm{SC}}(\mathrm{i}) \text {. }
$$

Figura 3 mostra o resultado do cálculo da intensidade de segregação relativa $I_{S C}(i)$ da impressão de Baumann da Figura 1 através das fórmulas (1) e (2). O objetivo da metodologia computacional desenvolvida é determinar o tipo e a espessura da linha de segregação conforme o padrão aplicado através da intensidade da segregação relativa.

Primeiramente, aparece-se mais óbvio, que o tipo (continuidade) pode ser determinado através da função $\mathrm{I}_{\mathrm{SC} \_\mathrm{R}}(\mathrm{i})$, menos a espessura da linha de segregação.

\footnotetext{
* Contribuição técnica ao 450 Seminário de Aciaria - Internacional, 25 a 28 de maio de 2014,
} 

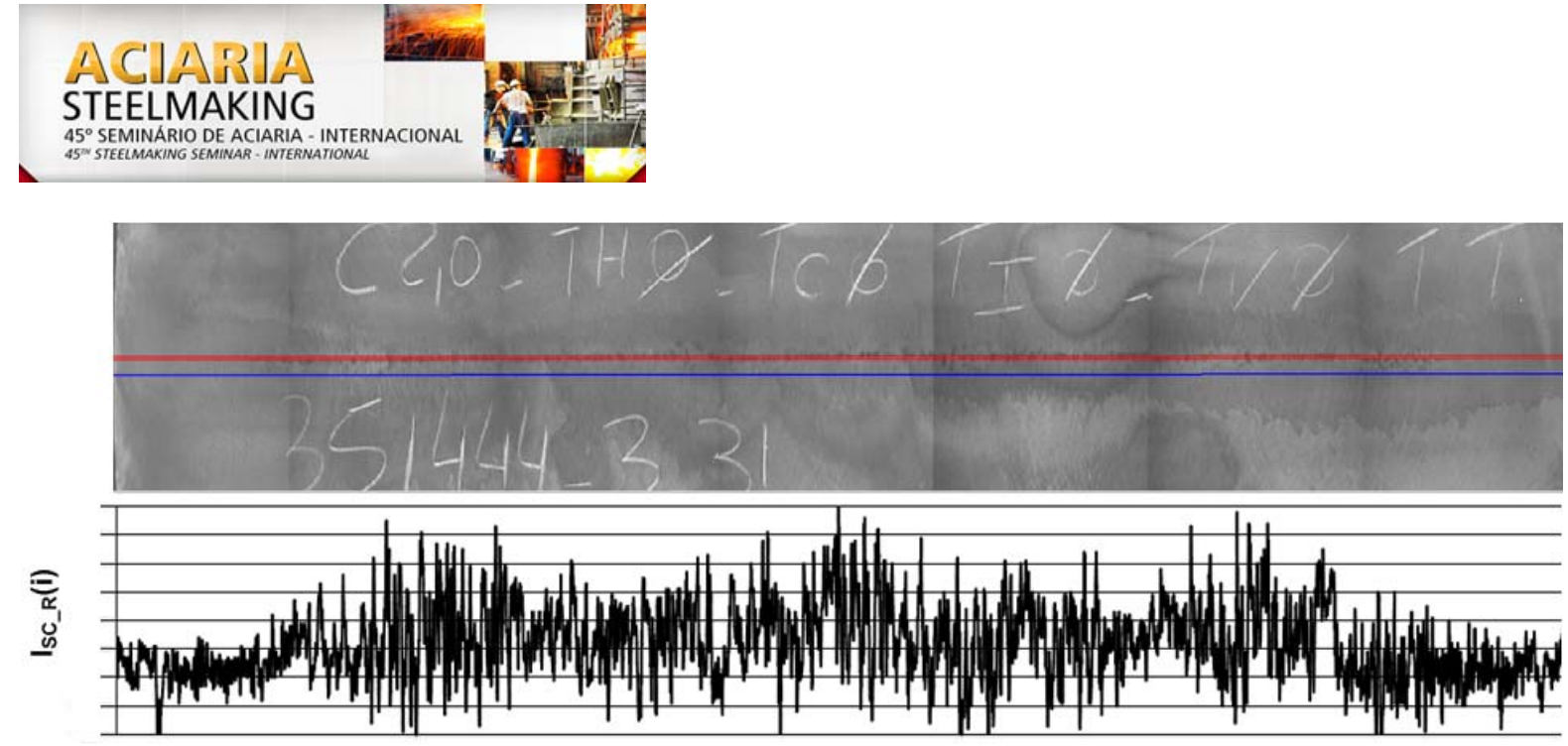

Figura 3. Resultado do cálculo da intensidade de segregação relativa $I_{S C_{-} \mathrm{R}}(\mathrm{i})$ da impressão de Baumann mostrada na Figura 1. A faixa de segregação é mostrado em vermelho e a linha de referência em azul.

Uma metodologia para calcular a espessura seria a obtenção da quantidade de pixels que apresentam uma intensidade pronunciada em comparação com a linha de referência, e multiplicar esta quantidade com a extensão física de um pixel. Mas verificou-se, que a intensidade da segregação corresponde bem com a espessura da segregação, pois não existe, no mesmo tempo, uma segregação muito fina e intensa, ou ao contrário, uma formação de segregação muito espessa e leve.

A avaliação poderia ser feito através de uma comparação das intensidades de segregação relativas conforme Figura 4.
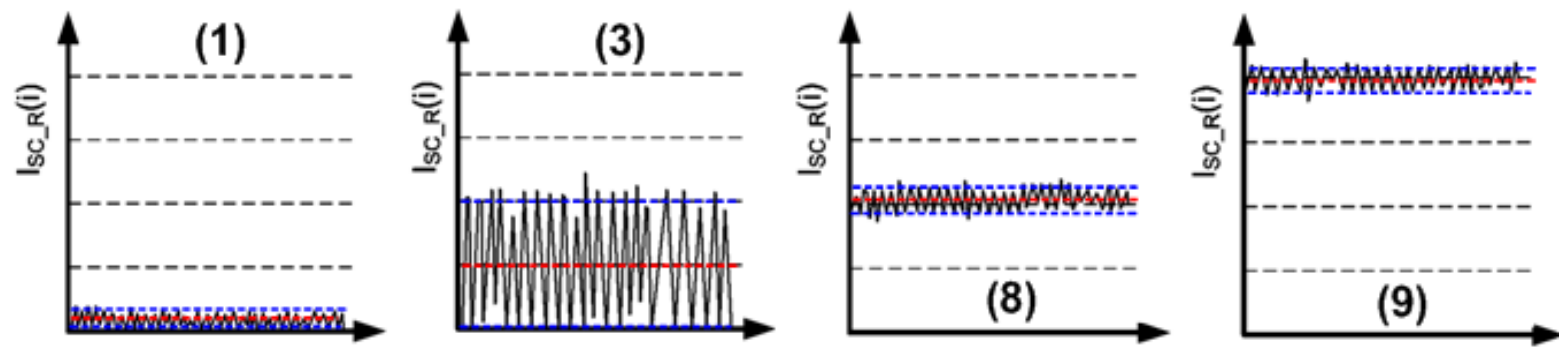

Figura 4. Representação esquemática das funções de intensidade de segregação relativas dos tipos de segregação relacionado com Tabela 1. Os valores médios são indicados por meio da linha vermelha e as variações por meio das linhas azuis.

Figura 4 mostra, que a função de intensidade de uma segregação menos crítica (mostrado p. ex. através das imagens (1) \& (3) da Tabela 1) está variando com mais ou menos igual ao valor médio desta função. O valor médio e a variação aumentam juntos com o índice da espessura da segregação.

Em caso de uma segregação mais crítica (mostrado p. ex. através das imagens (8) \& (9) da Tabela 1), a função de intensidade relativa permanece num nível elevado, praticamente sem qualquer variação. Baseado nesta observação pode-se resumir que os índices de segregação diferentes podem ser determinados através do valor médio e do desvio padrão da função da intensidade de segregação relativa. Será mostrado posteriormente, que esse comportamento do valor médio e do desvio padrão da função da intensidade de segregação relativa relacionada com uma segregação da criticidade média (mostrado p. ex. através das imagens (4) - (6) da Tabela 1) fica entre os comportamentos observados na Figura 4 . O valor médio $\overline{\mathrm{I}_{\mathrm{SC} \_\mathrm{R}}}(\mathrm{i})$ e o desvio padrão $\sigma_{\mathrm{SC}_{-} \mathrm{R}}(\mathrm{i})$ da função de intensidade da segregação relativa,

* Contribuição técnica ao 45 Seminário de Aciaria - Internacional, 25 a 28 de maio de 2014, Porto Alegre, RS, Brasil. 
$\mathrm{I}_{\mathrm{SC}} \mathrm{R}(\mathrm{i})$, de um ponto específico (i), são calculados utilizando uma série de valores $\mathrm{N}_{\mathrm{A}}, \mathrm{N}_{\mathrm{A}} / 2$ valores antes e após do ponto (i) respectivamente.

$$
\begin{aligned}
& \overline{\mathrm{I}_{\mathrm{SC}_{-} \mathrm{R}}}(\mathrm{i})=\frac{1}{\mathrm{~N}_{\mathrm{A}}} \sum_{\mathrm{j}=\mathrm{i}-\mathrm{N}_{\mathrm{A}} / 2}^{\mathrm{j}=\mathrm{i}+\mathrm{N}_{\mathrm{A}} / 2} \mathrm{I}_{\mathrm{SC}_{-} \mathrm{R}}(\mathrm{j}) \quad \text { se } \mathrm{N}_{\mathrm{A}} / 2 \leq \mathrm{j} \leq \mathrm{N}-\mathrm{N}_{\mathrm{A}} / 2 \\
& \overline{\mathrm{I}_{\mathrm{SC} \_\mathrm{R}}}(\mathrm{i})=0 \\
& \text { se } \mathrm{j}<\mathrm{N}_{\mathrm{A}} / 2 \text { ou } \mathrm{j}>\mathrm{N}-\mathrm{N}_{\mathrm{A}} / 2
\end{aligned}
$$

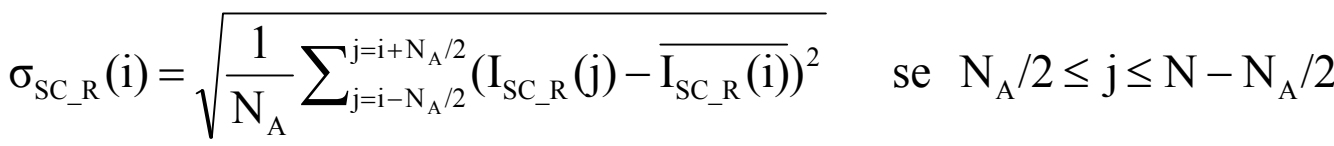

$$
\begin{aligned}
& \sigma_{\mathrm{SC} \_\mathrm{R}}(\mathrm{i})=0 \\
& \text { se } \mathrm{j}<\mathrm{N}_{\mathrm{A}} / 2 \text { ou } \mathrm{j}>\mathrm{N}-\mathrm{N}_{\mathrm{A}} / 2
\end{aligned}
$$

Figura 5 mostra o resultado do cálculo do valor médio, $\overline{\mathrm{I}_{\mathrm{SC} \_\mathrm{R}}}(\mathrm{i})$, e do desvio padrão, $\sigma_{\mathrm{SC} \_\mathrm{R}}(\mathrm{i})$, da impressão de Baumann da Figura 1 utilizando as fórmulas (3) e (4).

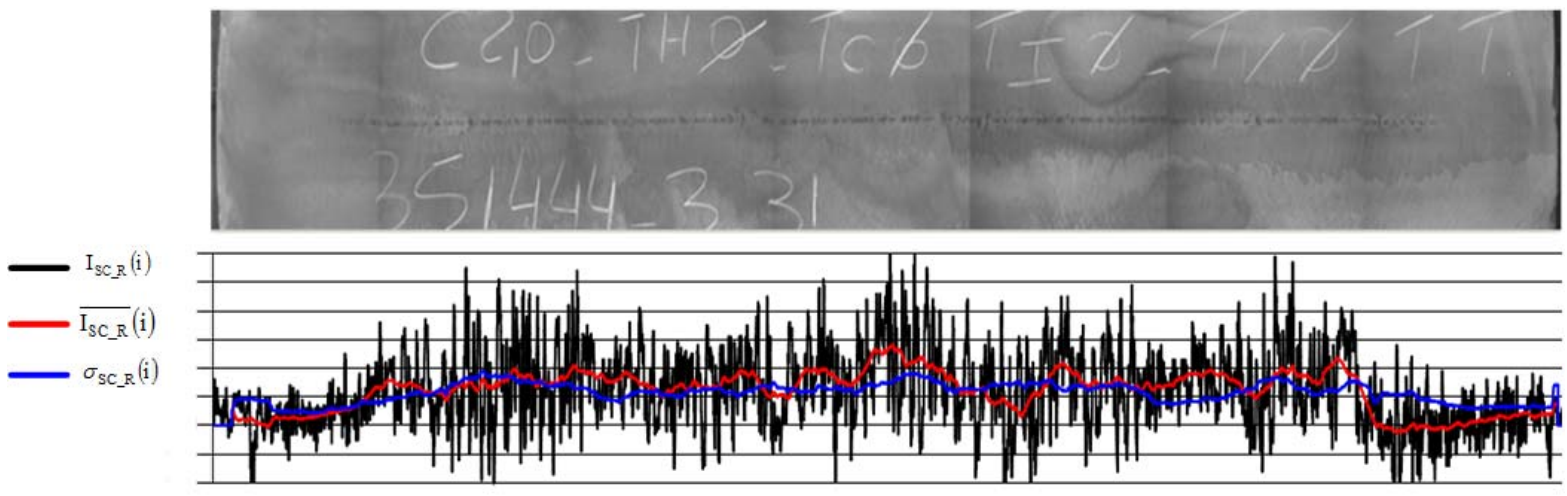

Figura 5. Resultado do cálculo do valor médio e o desvio padrão da função de intensidade da segregação relativa da impressão de Baumann mostrada na Figura 1.

Como já foi mostrado anteriormente através dos quatro casos específicos na Figura 4, os dois parâmetros, valor médio e desvio padrão da intensidade de segregação relativa, são conectados aos índices da segregação central (continuidade e a espessura). O mapeamento dos parâmetros foi feito pela análise de várias impressões de Baumann. O resultado desta análise é resumido num diagrama único na Figura 6, e em concordância da ideia principal conforme Figura 4. Observou-se na Figura 6, que os níveis de segregação são claramente separados em três áreas. A espessura de segregação aumenta com a distância da origem do diagrama.

A implementação prática do mapeamento dos índices da segregação, conforme Figura 6, foi realizado através de uma rede neural feedforward [14,15], treinado pelos pontos de dados mostrados na Figura 6 (valor médio e desvio padrão da intensidade de segregação, tipo e espessura de segregação). Geralmente, uma rede neural tem o poder de um aproximador universal. Ela pode realizar um mapeamento arbitrário de um espaço vetorial (dados de entrada) para um outro espaço vetorial (dados de saída).

\footnotetext{
* Contribuição técnica ao 45 Seminário de Aciaria - Internacional, 25 a 28 de maio de 2014,
} 

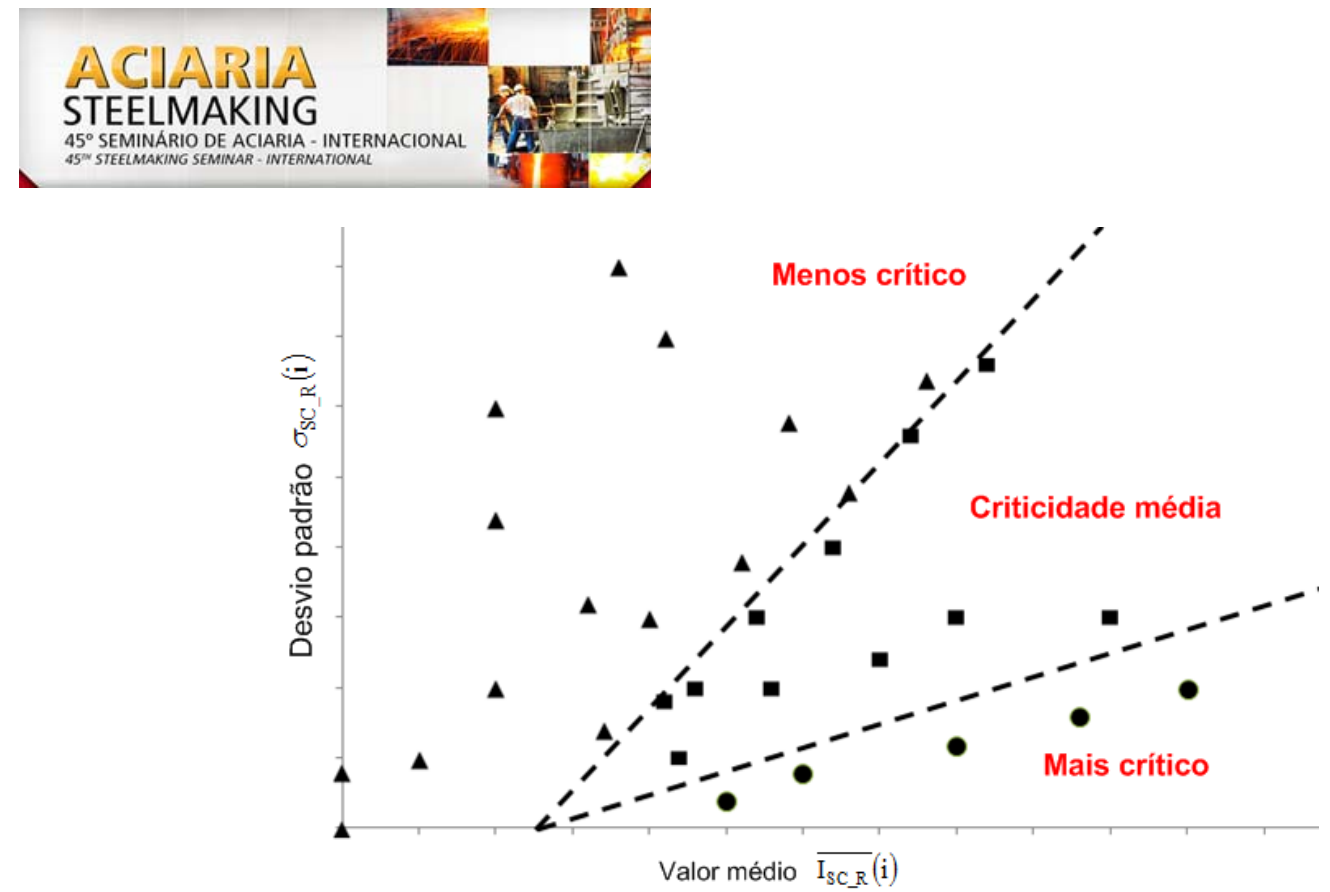

Figura 6. Mapeamento dos índices da segregação central com o valor médio e o desvio padrão da intensidade de segregação relativa.

A rede neural consiste de neurônios, que estão ordenados em camadas. Cada neurônio, numa determinada camada, está relacionado com todos os neurônios da próxima camada. Nas redes neurais feedforward, os dados sempre fluem estritamente da entrada à saída da rede, sempre sem conexões de retroalimentação. A primeira camada é chamada de camada de entrada, a última camada de camada de saída e as camadas intermédias de camadas ocultas. Para a aplicação específica da avaliação da segregação central, foi definida uma rede neural, conforme Figura 7, consistindo de dois neurônios de entrada (E1, E2: valor médio e desvio padrão da função de intensidade da segregação relativa), quatro neurônios ocultos em uma única camada oculta $(01-O 4)$, e dois neurônios de saída (S1, S2: tipo e espessura da segregação).

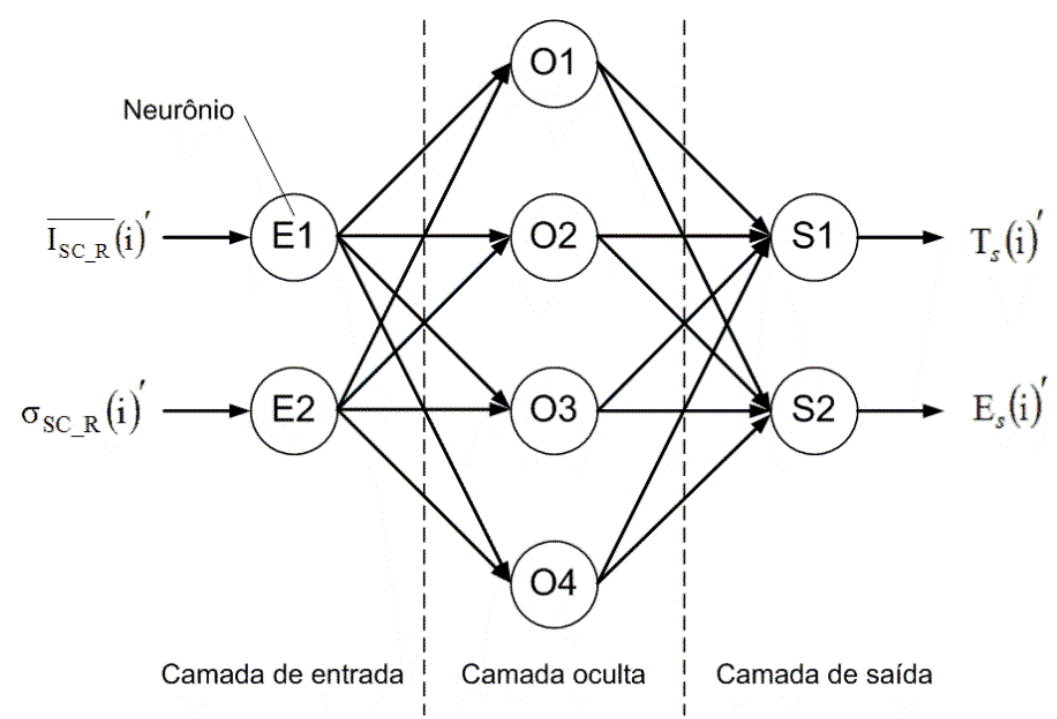

Figura 7. Estrutura da rede neural utilizada neste trabalho.

A conexão entre dois neurônios diferentes, $x_{i}$ e $x_{j}$, é caracterizada pelo coeficiente de peso $\omega_{\mathrm{ij}}$ e o coeficiente de bias $\vartheta_{\mathrm{i}}=1$ do neurônio i. O coeficiente de peso individual $\omega_{\mathrm{ij}}$ reflete o grau de importância desta conexão específica da rede neural. $\mathrm{O}$ valor de saída do neurônio $x_{i}$ é determinado pelas equações

* Contribuição técnica ao $45^{\circ}$ Seminário de Aciaria - Internacional, 25 a 28 de maio de 2014, Porto Alegre, RS, Brasil. 


$$
\begin{array}{r}
x_{i}=f\left(\xi_{i}\right) \\
\operatorname{com} \xi_{i}=\vartheta_{i}+\sum_{j} \omega_{i j} x_{j},
\end{array}
$$

onde $\xi_{\mathrm{i}}$ é o potencial do neurônio $x_{\mathrm{i}}$. A função $\mathrm{f}\left(\xi_{\mathrm{i}}\right)$ é chamada função de transferência ou função de ativação. Neste trabalho, a função do tipo "sigmoide",

$$
\mathrm{f}\left(\xi_{\mathrm{i}}\right)=\frac{1}{1+e^{-\xi_{i}}}
$$

foi escolhido como a função de transferência $f\left(\xi_{\mathrm{i}}\right)$. Em geral, uma rede neural apenas pode processar dados de entrada ou saída normalizados entre zero e um. Por isso, os valores de entrada (valor médio e desvio padrão da intensidade de segregação relativa) e os valores de saída (tipo e espessura da segregação central) foram transformados (escalados) para valores adequados entre zero e um. Os valores de entrada foram divídidos com o máximo individual:

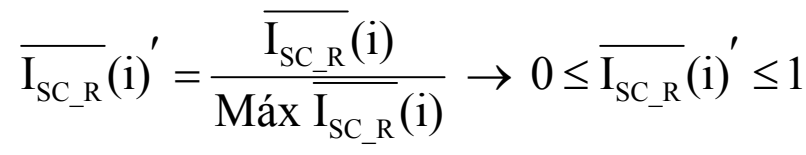

$$
\begin{aligned}
& \sigma_{\mathrm{SC}_{-} \mathrm{R}}(\mathrm{i})^{\prime}=\frac{\sigma_{\mathrm{SC} \_\mathrm{R}}(\mathrm{i})}{\operatorname{Máx}\left(\sigma_{\mathrm{SC} \__{-}}(\mathrm{i})\right)} \rightarrow 0 \leq \sigma_{\mathrm{SC}_{-} \mathrm{R}}(\mathrm{i})^{\prime} \leq 1 \text {. }
\end{aligned}
$$

Para escalar os valores de saída, os três tipos de segregação diferentes foram transformados para valores numéricos $\left(\mathrm{T}_{S}(\mathrm{i})^{\prime}\right), 0,0,5$ e 1 , respectivamente. Da mesma maneira, as espessuras de segregação diferentes foram transformadas para valores numéricos $\left(E_{S}(i)^{\prime}\right)$ entre 0 e 1.

O algoritmo de aprendizagem supervisionada largamente utilizados é de retropropagação. A ideia é inicializar primeiramente todos os coeficientes de peso com valores aleatórios e, iterativamente, inserir um conjunto de valores de entrada (Figura 6) na rede neural e processá-lo, camada a camada, até obter os dois valores de saída (tipo e espessura da segregação). O erro médio quadrático entre os valores de saída objetivados e os valores de saída obtidos pelo cálculo:

$$
E=\frac{\sqrt{\left(\mathrm{T}_{\mathrm{S}}{ }^{\prime}-\mathrm{T}_{\mathrm{S} \text { obj }}^{\prime}\right)^{2}+\left(\mathrm{E}_{\mathrm{S}}{ }^{\prime}-\mathrm{E}_{\mathrm{S} \text { obj }}{ }^{\prime}\right)^{2}}}{2}
$$

é determinado e propagado no sentido contrário da rede neural para ajustar os coeficientes de pesos individuais. A retropropagação foi implementada segundo a filosofia do gradiente descendente,

$$
\overrightarrow{\omega_{\mathrm{ij}_{\mathrm{k}}}}=\overrightarrow{\omega_{\mathrm{ij}_{\mathrm{k}-1}}}-\lambda \nabla \mathrm{E}
$$

onde $\overrightarrow{\omega_{\mathrm{ij}_{\mathrm{k}}}}$ representa o vetor de pesos, $\nabla \mathrm{E}$ o gradiente do erro médio quadrático, $\lambda$ um fator de aprendizagem, que foi escolhido adequadamente, e k o índice do passo de iteração atual. Desta forma, o algoritmo modifica o vetor de pesos $\overrightarrow{\omega_{\mathrm{ij}_{\mathrm{k}}}}$, sempre na direção contrária à direção de maior aumento do erro médio quadrático, e minimiza o erro médio quadrático até a rede funcionar como desejada conforme Figura 6.

* Contribuição técnica ao $45^{\circ}$ Seminário de Aciaria - Internacional, 25 a 28 de maio de 2014, Porto Alegre, RS, Brasil. 


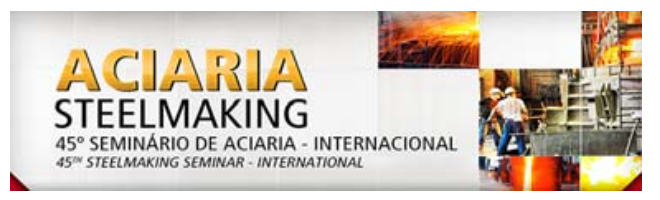

Neste trabalho, o processo iterativo foi interrompido após o erro médio quadrático atingir um valor menor do que 0,001, o que levou cerca de 50000 iterações. Depois do treinamento, a rede neural ficou pronta para a aplicação no processo de avaliação da segregação central. A Figura 8 mostra o resultado da avaliação da impressão de Baumann da Figura 1 com a utilização da rede neural treinado.

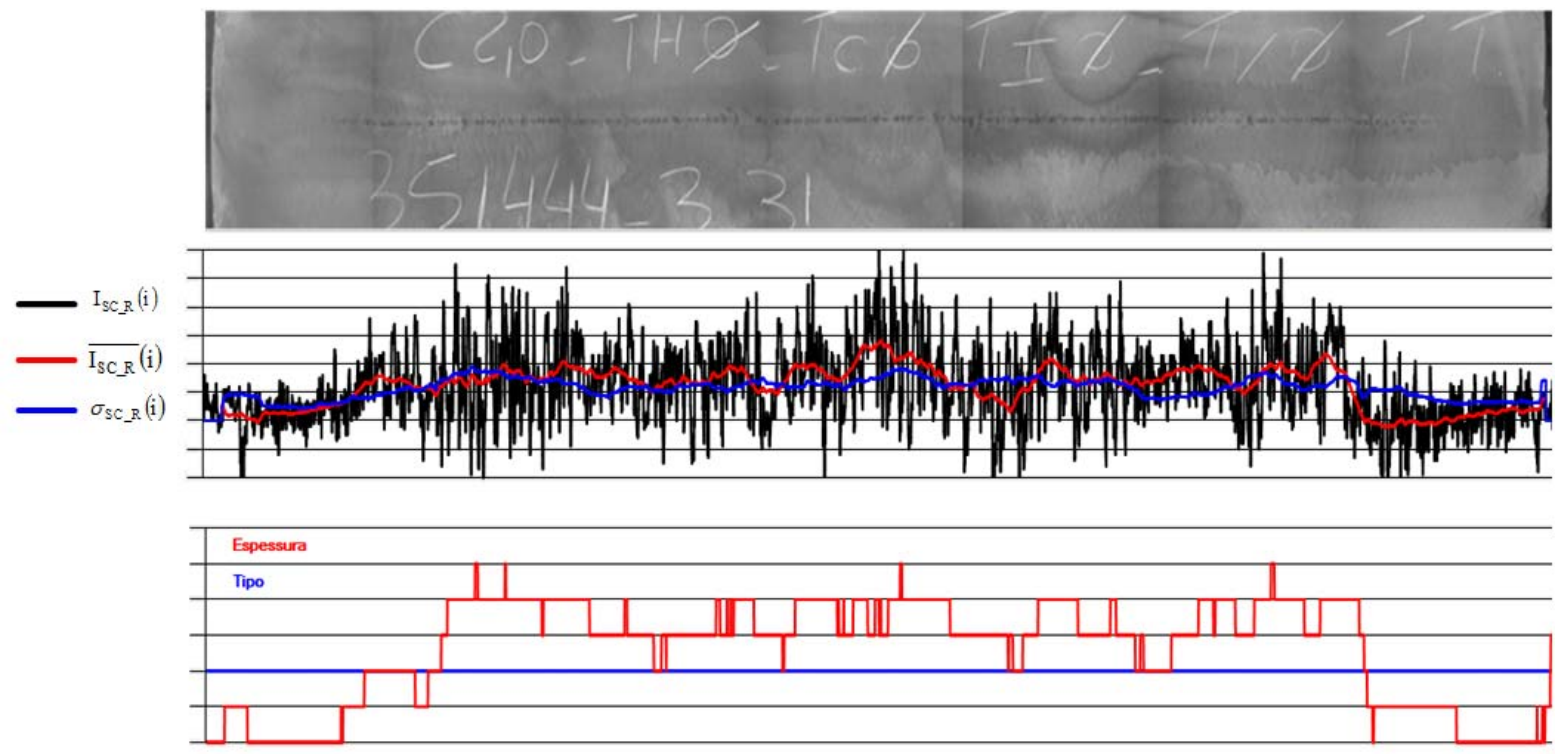

Figura 8. Resultado da avaliação da impressão de Baumann da Figura 1 utilizando a rede neural. No segundo diagrama, a distribuição do tipo de segregação é indicada por meio da linha azul e da espessura da segregação por meio da linha vermelha.

Pode ser visto na Figura 8, que neste exemplo, que o tipo de segregação (linha azul) permanece constante e a espessura de segregação (linha vermelha) varia ao longo da largura da amostra.

O último passo da avaliação computacional é a determinação do índice de segregação final, válido para a amostra inteira. $O$ índice final é determinado em geral pelo pior índice de segregação da amostra, mas foi definido que o índice tem que ter, no mínimo, uma extensão significativa de $3 \%$ da largura total da placa. Em caso de uma extensão menor do que 3\% da largura, o próximo pior índice será escolhido. Este procedimento é repetido até a soma das extensões dos índices específicos sendo maior do que a extensão mínima. Na Figura 8, os quatro picos que indicam a maior espessura da segregação central, não contam para o resultado final, pois a extensão total delas é menor do que $3 \%$ da largura total da placa. Por isso, 0 próximo pior índice foi determinado como o resultado final, e corresponde ao resultado da avaliação manual do especialista do laboratório de Baumann.

\section{RESULTADOS}

A metodologia da avaliação deste trabalho foi implementado em um programa independente, com acesso direto ás imagens das impressões de Baumann. A Figura 9 mostra uma captura de tela deste programa.

* Contribuição técnica ao 450 Seminário de Aciaria - Internacional, 25 a 28 de maio de 2014, 

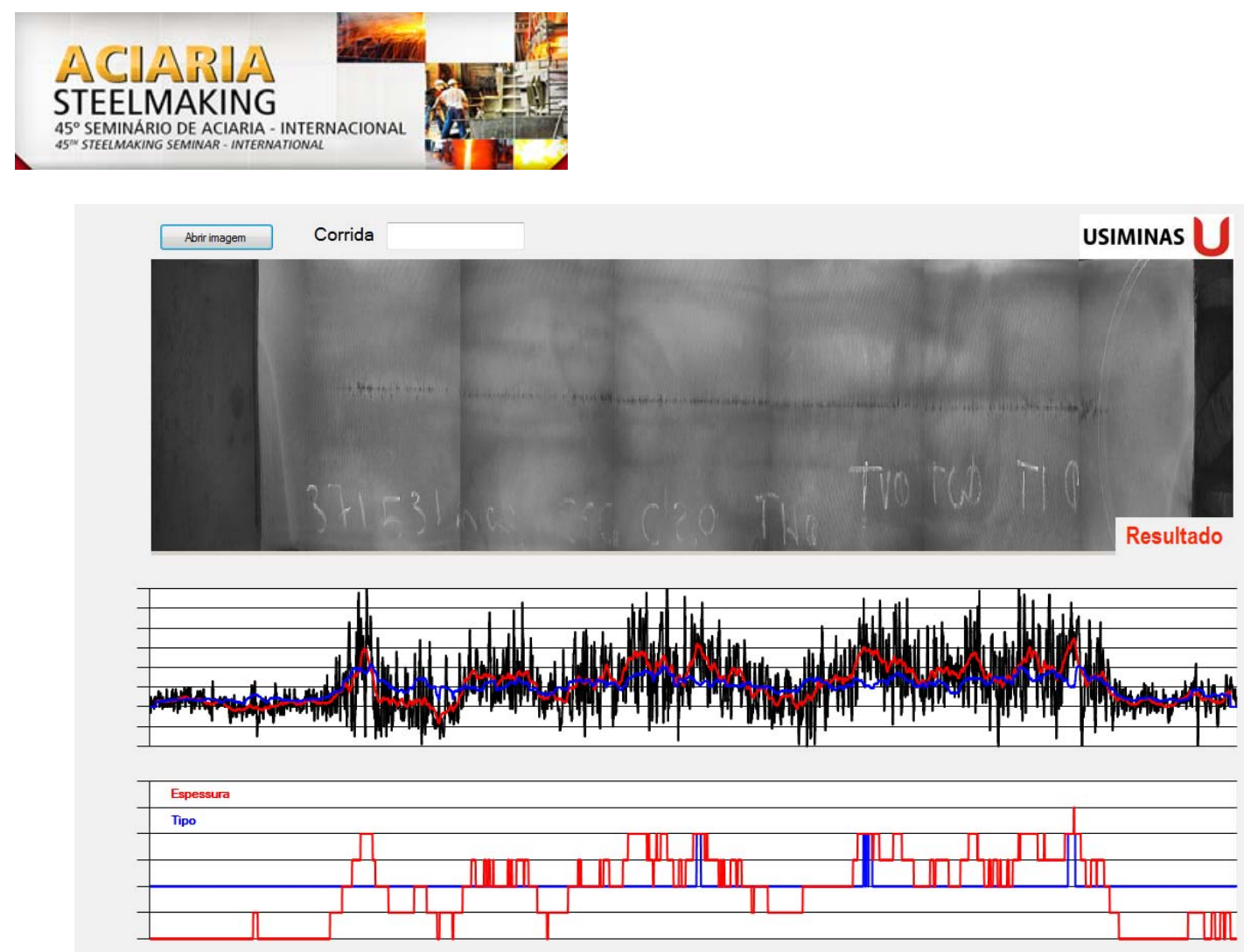

Figura 9. Captura de tela do programa de análise da aciaria da Usiminas.

O programa de análise oferece, além da metodologia apresentada, características adicionais como a pesquisa de imagens pelo número de corrida e a detecção automática da linha de segregação na imagem (indicada por meio das linhas amarelas na Figura 10). O resultado final da análise é indicado no canto inferior direito da imagem e, finalmente, armazenado junto com informações adicionais (data da avaliação e número da amostra) em um banco de dados para possibilitar uma avaliação posterior dos resultados.

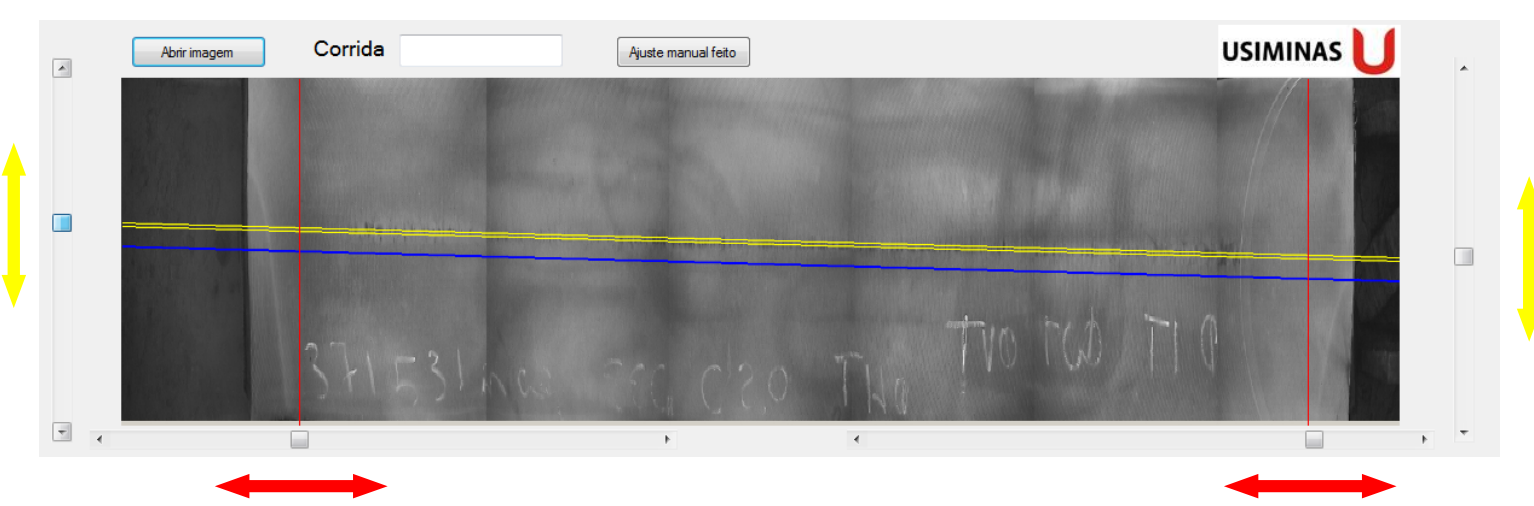

Figura 10. Ajuste manual da faixa de segregação e limitação do intervalo de análise com barras laterais.

Se, por problemas relativo a qualidade da imagem, a detecção automática da linha central não tiver sucesso, um ajustamento manual da faixa da segregação central ainda pode ser feito através das barras laterais aos ambos os lados da imagem da amostra (indicadas por meio das setas amarelas na Figura 10).

O intervalo da avaliação ao longo da largura da placa pode ser limitado através das barras horizontais abaixo da imagem (indicadas por meio das setas vermelhas na Figura 10). Uma limitação do intervalo de avaliação é necessária se a imagem tiver uma extensão maior do que a largura da placa (isso gera barras escuras aos lados da imagem), uma análise de uma região de imagem específica não fizer sentido ou

* Contribuição técnica ao 450 Seminário de Aciaria - Internacional, 25 a 28 de maio de 2014, Porto Alegre, RS, Brasil. 
se der resultados errados devido às deficiências na preparação da impressão de Baumann (p. ex. uma deficiência no corte ou na usinagem da amostra). Este programa de avaliação das impressões de Baumann está em uso pleno na aciaria da Usiminas desde setembro 2013.

\section{DISCUSSÃO}

A ideia da avaliação da espessura de segregação através da intensidade de segregação provou ser uma metodologia confiável. A Figura 11 mostra um exemplo ilustrativo da correlação proporcional entre a intensidade e a espessura da segregação por meio de um trecho de uma amostra que apresentou uma segregação central muito pronunciada.

(a)

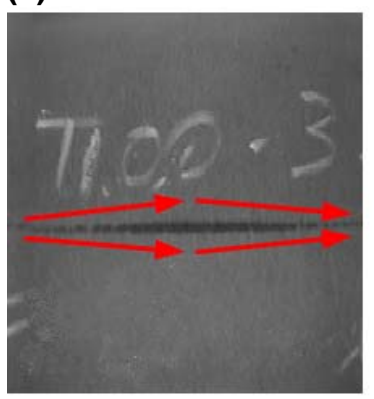

(b)

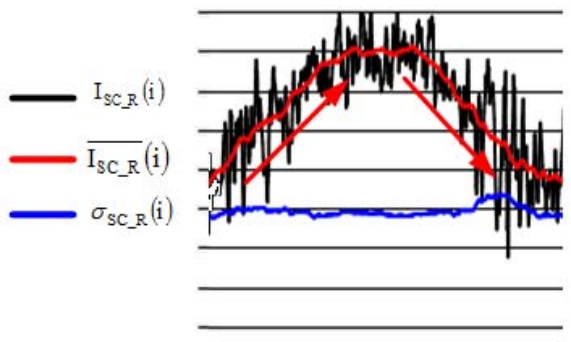

(c)

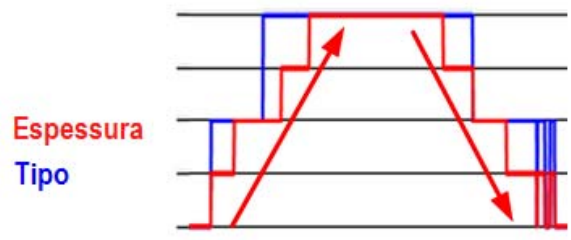

Figura 11. Correlação proporcional entre a espessura de segregação física (a) e a intensidade de segregação (b); Resultado da avaliação computacional (c).

A Figura 11a mostra, que a segregação deste trecho aumenta rapidamente da segregação leve para segregação severa e volta novamente à segregação leve. A correlação proporcional entre a espessura e a intensidade de segregação pode ser observada pela comparação das Figuras $11 \mathrm{a}$ e 11b. O valor médio da intensidade de segregação (indicada por meio da linha vermelha na Figura 11b) sobe e desce proporcionalmente com a espessura da segregação física observada. O desvio padrão da intensidade de segregação (indicada por meio da linha azul na Figura 11b) permanece em um valor constante durante transição da segregação. Figura 11c mostra a avaliação da segregação computacional do trecho da amostra, utilizando o algoritmo desenvolvido neste trabalho.

$\mathrm{Na}$ Figura 12, os resultados da avaliação manual são comparados com os resultados do programa computacional.

(a)

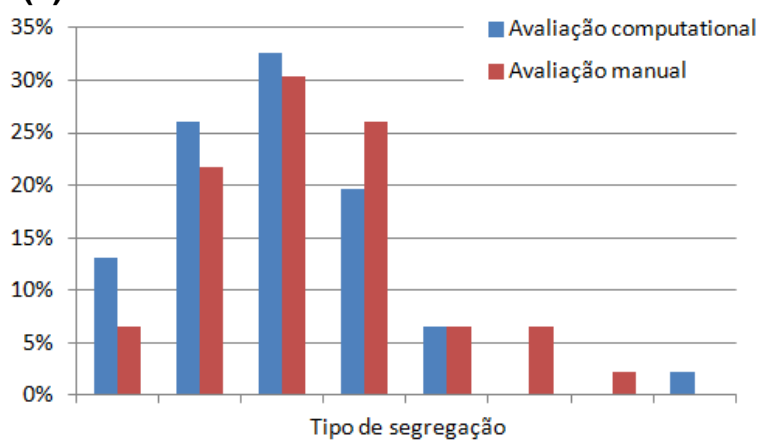

(b)

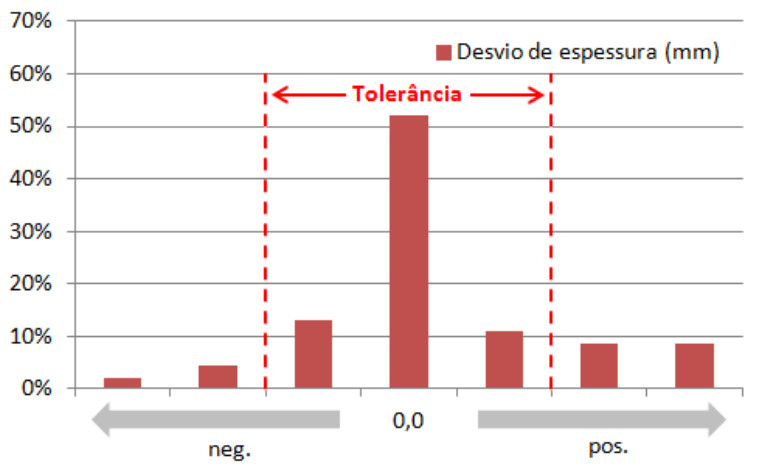

Figura 12. Comparação dos resultados finais (a) e desvios de intensidade (b) das avaliações manuais e computacionais utilizando a metodologia apresentado neste trabalho.

* Contribuição técnica ao 450 Seminário de Aciaria - Internacional, 25 a 28 de maio de 2014, 
Os resultados representam um total de 46 amostras de placas da máquina de lingotamento contínuo 3 de Cubatão, que foram obtidas durante um mês de produção. A Figura 12a, a distribuição dos tipos de segregação destas amostras, mostra uma boa concordância entre as avaliações manuais e computacionais. A continuidade foi avaliada com $100 \%$ de concordância. Diferenças podem ser observadas nas espessuras de segregação.

Uma visão geral da distribuição do desvio de espessura entre as duas metodologias de avaliação é apresentada na Figura 12b. Uma diferença tolerável da espessura da segregação foi, conforme a norma interna, atingida em $76 \%$ dos casos, o que é um bom resultado e mostra a boa concordância entre as avaliações. Os casos que excederam a tolerância interna foram investigados separadamente, resultando numa melhoria dos equipamentos e do processo da preparação das amostras.

\section{CONCLUSÃO}

No presente trabalho, uma metodologia de avaliação computacional das impressões de Baumann foi desenvolvida. A determinação computacional do índice de segregação através do processamento digital das imagens em tons de cinza baseiase no valor médio e o desvio padrão da intensidade de segregação em comparação com a intensidade fora da faixa da segregação central. O mapeamento dos índices de segregação com o valor médio e o desvio padrão da intensidade relativa segregação foi realizado com a utilização de uma rede neural feedforward.

Com a introdução deste sistema novo ao processo de produção na aciaria em Cubatão, conseguimos estabelecer uma base de monitoramento da segregação central padronizada e confiável. Através desta base, os parâmetros de processo de lingotamento contínuo estão otimizados sucessivamente, com o objetivo principal em monitorar e melhorar a qualidade interna das placas e os produtos laminados.

A rotina está implementada e inserida no Sistema de Gestão da Qualidade.

Por se tratar de uma nova tecnologia, que demandou atividade inventiva em seu desenvolvimento e com potencial para comercialização, foi solicitada a proteção legal através de patente.

\section{REFERÊNCIAS}

1 Flemings MC. Solidification processing. New York: McGraw Hill; 1974.

2 Ayata K, MORI H, Taniguchi K, Matsuda H. Low Superheat Teeming with Electromagnetic Stirring. ISIJ Int. 1995; 35(6):680-685.

3 Chang-Hee Y, Young-Mok W. On-line internal quality prediction technology of slab for heavy plates. La Revue de Métallurgie. 2008;105(7-8):375-382.

4 Sun Q, Yu G, Zhu Z. Study on the Relationship between the Cooling Condition in Width and the Centerline Segregation of the Slab. In: AISTech Conference Proceedings; 2009. St. Louis: AIST; 2009. v.1, p.1099-1110.

5 Miyazawa K, Schwerdtfeger K. Macrosegregation in continuously cast steel slabs preliminary theoretical investigation on the effect of steady state bulging. Arch. Eisenhüttenw. 1981;52(11):415-422.

6 Mörwald K, Thalhammer M, Federspiel C, Gould L. Improvements in HIC Resistance with ASTC. In: $4^{\text {th }}$ European Continuous Casting Conference; 1994, Birmingham, Reino Unido. Birmingham: IOM Communications; 2002. ref. C0208/80.

7 Chen X, Abraham S, Franco G, Asante J, D'souza C, Dunnet K. Improving Centerline Segregation Control on X70 Products. In: AISTech Conference Proceedings; 2010, Pittsburgh, EUA. Pittsburgh: AIST; 2010. v.2, p.175-180.

\footnotetext{
* Contribuição técnica ao 45 Seminário de Aciaria - Internacional, 25 a 28 de maio de 2014, 
8 Vander Voort GF. Metallography: Principles and Practice (\#06785G). Materials Park: ASM International, 1984.

9 Jacobi HF. Investigation of Centreline Segregation and centreline porosity in CC. Steel Research. 2003;74(11-12):667-678.

10 International Standard Organization. ISO 4968: Steel - Macrographic examination by sulphur print (Baumann method). Geneva; 1979.

11 British Standard Institution. BS 6285 - The macrographic examination of steel by sulphur print (Baumann method). Londres; 1982.

12 American Society for Testing and Materials. ASTM E 1180-08 - Standard practice for preparing sulphur prints for macrostructural evaluation. West Conshohocken; 2008.

13 Sancho LF, Díaz AM, Sirgo JA. New technique for centre line segregation measurement in steel slabs. La Revue de Métallurgie. 2006;103(12): 546-551.

14 Rojas R. Neural Networks - A Systematic Introduction. Berlin: Springer; 1996.

15 Rumelhart DE., Mcclelland JL. Parallel distributed processing: explorations in the microstructure of cognition. Cambridge: MIT Press; 1986.

* Contribuição técnica ao 450 Seminário de Aciaria - Internacional, 25 a 28 de maio de 2014, Porto Alegre, RS, Brasil. 